\title{
Aprendizaje basado en PROBlemas y PROYeCtos DE INVESTIGACIÓN: HACIA UN PENSAMIENTO COMPLEJO
}

\author{
LEARNING BASED IN PROBLEMS AND INVESTIGATIVE PROJECTS: \\ GOING TO A COMPLEX THINKING
}

\author{
MSc. René Cortijo Jacomino \\ Universidad Tecnológica Israel \\ rcortijo@uisrael.edu.ec \\ Fecha de envío del artículo: 06/05/2014 \\ Fecha de aceptación del artículo: 25/06/2014
}

\section{Resumen:}

Cuando analizamos el campo laboral real al que se enfrentarán los profesionales recién graduados, nos encontramos con la necesidad de que estos estudiantes de educación superior estén capacitados para enfrentar y resolver problemas de pensamiento complejo. Partiendo de esta idea se plantea el ABP: Aprendizaje basado en problemas, como eje integrador del contenido a estudiar; teniendo como idea central conducir el aprendizaje de problema en problema, articulando ideas y proyectos.

Cuando el estudiante se encuentra enfrentando y solucionando diferentes tipos de problemas con complejidad creciente esto le acerca a competencias proyectadas en cualquier nivel educacional.

Palabras clave: Aprendizaje, Complejidad, Problemas, Resolución, Investigación, Estudiantes 


\section{Abstract:}

When we analyze the real career field to what recently graduate professionals have to face, we find ourselves with the need of that these superior education students have to be trained to face and resolve complex thinking problems. From this idea it arises the PBL: Problem-based learning, as an integrator axe for the content to be studied; having as a central idea to steer the knowledge problem to problem, articulating projects and ideas.

When the student finds himself facing and resolving different types of problems with growing complexity it gets them closer to projected competencies in any educational level.

Keywords: Learning, Complexity, Problems, Resolution, Investigation, Students 


\section{Aprendizaje basado en problemas y proyectos de investigación: hacia un pensamiento complejo}

La educación superior debe generar en los estudiantes competencias para pensar y crear, a través de la resolución de problemas y la realización de proyectos de investigación dentro del proceso de formación profesional, en una perspectiva cognitivista y constructivista del aprendizaje.

El mundo del trabajo requiere profesionales capacitados para enfrentar y resolver problemas de complejidad progresiva con diferentes variables y donde intervienen simultáneamente diferente ciencias. Por otro lado, las tecnologías y herramientas del quehacer profesional varían con mucha rapidez generando cambios significativos en lo económico y social. Esas capacidades hay que desarrollarlas en todo el proceso de formación: desde cada una de la materias de estudio, proyectos y periodos de prácticas a lo largo de toda la carrera.

La idea central del proceso educativo es entonces conducir el aprendizaje de problema en problema, con la articulación de tareas y proyectos de investigación; esto significa generar una sistematización de problemas por etapas de estudio, para promover diversos resultados de aprendizaje.

Un programa de estudio se descompone en diversas asignaturas o módulos de estudio y estas, a su vez, en unidades temáticas con un sistema de actividades docentes: clases, prácticas de laboratorio, talleres, trabajo en áreas de producción, trabajos investigativos, y otras que se proyectarán sobre la base de problemas, tanto reales como simulados de acuerdo con las condiciones existentes e intereses didácticos.

\section{ABP: aprendizaje basado en problemas}

El ABP como eje dinamizador e integrador del contenido a estudiar, ofrece un conjunto de posibilidades de orden psicológico y pedagógico, entre las que se encuentran:

- Enfrenta al sujeto con la realidad, la reconoce y adopta una postura frente a ella.

- Provoca una mayor actividad en los estudiantes, al intentar estos resolver la situación, al buscar respuestas científicas y tecnológicas en la realidad, al aprender a modificarla. 
- Favorece la formación de un pensamiento creativo, cuando el estudiante trata de hallar soluciones desde lo diferente: al encontrar y establecer relaciones, consolidaciones y respuestas lógicas.

- Propicia la permanente duda, una actitud positiva hacia la investigación científica como modo de abordar la realidad y de llegar a resultados objetivos en su perfeccionamiento.

- Contribuye a romper con el aprendizaje fraccionado, atomizado: integra y sistematiza conocimientos y habilidades.

- Desarrolla un mayor interés por la profesión, al promover un aprendizaje significativo donde se manifiestan sentimientos de pertenencia, de satisfacción por la labor realizada.

No obstante estas posibilidades mencionadas, el ABP porta contradicciones y exigencias que deben ser valoradas con profundidad, a fin de concebir una adecuada proyección metodológica para el proceso de enseñanza-aprendizaje. La contradicción es la decisión en cuanto a si se desarrolla el contenido de los programas educativos de acuerdo con la "lógica del problema" o continúa predominando la "lógica de las ciencias". Lo más aconsejable es desarrollar las experiencias de aprendizaje dentro de una "lógica de compromiso", entre la sistematización que demandan las ciencias y exigencias de conocimientos que la realidad impone en un problema.

\section{La proyección de los problemas para guiar los aprendizajes}

Desde la perspectiva de la Pedagogía Crítica el "problema es la realidad, es la vida", por lo que él constituye el punto de partida para el desarrollo de las experiencias de aprendizaje. Del problema se derivan el objetivo a lograr en las actividades educativas, los conocimientos y habilidades a desarrollar; se determinan el producto final a lograr en el aprendizaje, los métodos y los procesos de trabajo que dan lugar al producto final, así como la estrategia que guiará el proceso de evaluación del estudiante.

En la proyección de los problemas de los programas educativos (Cortijo, 1995) se deben considerar las siguientes alternativas:

Problemas propedéuticos: dirigidos a solucionar insuficiencias de orden teórico práctico para enfrentar otros problemas con mayor grado de dificultad y nivel educativo.

Problemas situacionales: orientados a resolver situaciones, casos específicos de los procesos de la vida empresarial y social en general, como pueden ser: la rectificación de una o varias deficiencias de una determinada actividad, la reparación o ajuste de un sistema tecnológico, la elaboración de una planificación y otros.

Problemas proyectivos: encaminados a ofrecer una solución a las insuficiencias de mayor complejidad y que exigen un alto nivel de integración de conocimientos y habilidades; ellos deben producir algún aporte de orden teórico o práctico, como pueden ser: el perfeccionamiento de un sistema organizacional 
o sistema tecnológico, el diseño de nuevos objetos, productos o procesos de trabajo para la actividad profesional y otros.

El enfrentamiento y solución de diferentes tipos de problemas con complejidad creciente van acercando al estudiante a las competencias de actuación proyectadas en cualquier nivel educacional.

A partir de un problema propedéutico se desprende la presentación y sistematización, al menos, de un método de trabajo propio del área del saber que se trate el microcurrículo, asociadas a nuevos conceptos objeto de estudio; un problema situacional requiere la aplicación de varios métodos de trabajo con la integración de conceptos ya estudiados y nuevos fundamentos teóricos; mientras que un problema proyectivo permite sistematizar los diferentes métodos de trabajo estudiados con la integración de todo o gran parte de la base conceptual correspondiente.

Los estudiantes al enfrentarse a los problemas y encontrarles solución, deben pasar por diferentes procesos, sistematizando métodos de investigación, como son:

- Identificar los problemas y sus causas, llegando a su detección y diagnóstico.

- Determinar los métodos, vías y alternativas de solución. Seleccionar la mejor alternativa.

- Planificar, organizar y ejecutar, con destreza y racionalidad, las tareas prácticas que conducen a la solución del problema.

- Controlar y evaluar los resultados, así como las vías empleadas en el proceso de ejecución.

La competencia, en la solución de determinados "tipos de problemas", se va logrando en la medida en que el estudiante se enfrente sistemáticamente a variadas situaciones que generen la aplicación de estos procesos de trabajo, y que finalmente, se precise el modelo general de solución para el tipo de problema en cuestión.

Los equipos de profesores organizados por disciplinas de estudio, en análisis colegiado, deberán buscarle una solución adecuada a la estructuración del contenido en correspondencia con los requerimientos que impone el problema a resolver, sin descuidar el rigor científico que exige el mundo contemporáneo.

\section{Procesos de aprendizaje derivados de la solución de problemas}

La solución de problemas requiere un conjunto de procesos de aprendizaje que deben proyectarse y concentrarse desde cada una de las áreas del conocimiento, como son:

- El procesamiento de información científica y cultural;

- La experiencia en laboratorios;

- La construcción de conceptos;

- La estructuración de métodos de trabajo; 
- La fundamentación de criterios y argumentación oral;

- El trabajo directo en escenarios reales y virtuales.

Estos procesos -en su accionar práctico- suponen un sistema de "problemas-productos del aprendizaje y de proyectos de investigación", que van guiando el desarrollo de conocimientos y habilidades con una concepción articuladas e integradora, promoviendo diferentes exigencias la expresión del pensamiento y modo de actuar.

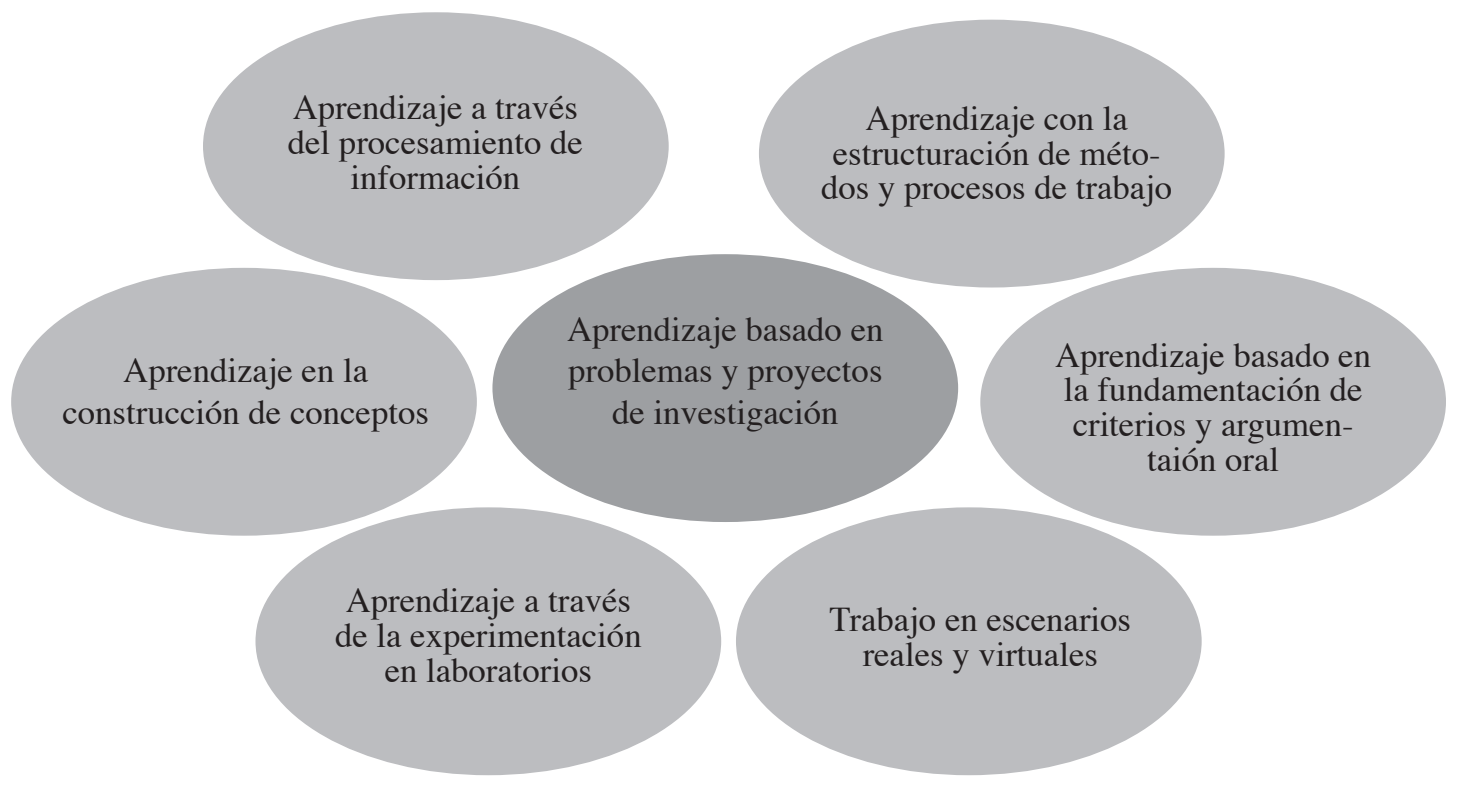

Elaborado por: Mg. René Cortijo Jacomino.

\section{La investigación formativa}

La investigación formativa debe construir el eje central del proceso de formación profesional, en función de la solución de problemas a través de la aplicación de los métodos de la investigación y métodos propios de las ciencias, deberá estar presente:

- Desde en el contenido de las asignaturas;

- La realización de proyectos de investigación curricular por etapas de formación;

- En la ejecución del proyecto de grado en opción al título profesional. 


\section{INVESTIGACIÓN FORMATIVA}

\section{Productos del aprendizaje en las asignaturas \\ 4 Proyectos de investigación por etapas de formación \\ 4 Proyectos en opción al grado académico}

Elaborado por: Mg. René Cortijo Jacomino

Las acciones investigativas deben dar lugar a procesos de:

- Búsqueda y procesamiento de información científica y cultural, en general;

- La aplicación del sistema conceptual de las diferentes asignaturas;

- La integración de las diferentes áreas del conocimiento;

- El descubrimiento de las alternativas de solución de los problemas;

- Productos concretos en orden teóricos y prácticos.

Es importante entonces que se planifique el sistema de productos del aprendizaje derivados de procesos investigativos, los que deberán ganar en complejidad de forma progresiva en función de las competencias profesionales a lograr.

Los productos del aprendizaje deberán dar lugar a:

- La elaboración de resúmenes de lecturas con identidad propia;

- La construcción de conceptos a partir de lecturas de varios autores y comprobaciones prácticas en la vida real;

- La aplicación de los métodos teóricos y prácticos de la investigación científica;

- El planteamiento y comprobación de hipótesis de quehacer científico, tecnológico, económico y social;

- La producción escrita, ponencias para foros-debates, ensayos y monografías;

- La defensa oral de fundamentos teóricos y criterios;

- El análisis y solución de casos de la vida real;

- La aplicación de modelos matemáticos y físicos;

- La aplicación de los procesos de trabajo que se emplean en la diferentes áreas del conocimiento de la carrera;

- El empleo de herramientas tecnológicas de gestión y tecnologías de la información y la comunicación, de forma general. 


\section{La evaluación del aprendizaje sobre la investigación formativa}

Se concibe la evolución de los resultados del aprendizaje como un proceso acumulativo e integrador, donde se valoran los avances y limitaciones de los estudiantes para controlar el accionar educativo; a la vez, que permite diagnosticar y reorientar el trabajo docente en función de la máxima calidad.

La evaluación del aprendizaje desde todas las materias de estudio y, en especial, de los proyectos de investigación constituyen la vía esencial para valorar en qué medida se van logrando las competencias por etapas de formación y las competencias de perfil del egresado, tanto genéricas como específicas.

Los eventos de evaluación del aprendizaje en función de las competencias siempre demandarán conocimientos y modos de actuación integradores en complejidad creciente en la medida que el estudiante avanza en el proceso de formación profesional. Las capacidades para investigar y crear tendrán una alta prioridad entre las exigencias de estándares e indicadores de calidad del proceso educativo. 


\section{Bibliografía}

Álvarez de Zayas, Carlos M. (2001). El Diseño Curricular. Educación y Desarrollo. La Habana, Cuba: Pueblo y Educación.

Ausbel David P., Novak joseph D y Hanesian Helen. (1996) Psicología Educativa. Un punto de vista cognoscitivo. México: Trillas.

Bruner Jerome S. (2001). El proceso mental en el aprendizaje. España: Nancea.

Cortijo Jacomino, René. (2002). Aprendizaje para investigar-crear. Quito, Ecuador: Klendarios.

Cortijo Jacomino, René. (2007). Modelo Curricular por competencias y proyectos. Quito, Ecuador: Klendarios.

Díaz Barriga, Frida y Hernández Rojas Gerardo. (2003). Estrategias docentes para un aprendizaje significativo. México: McGraw Hill.

Gonzales, Maura, Viviana y otros. (1995). Psicología para Educadores. La Habana, Cuba: Pueblo y Educación.

, (2003). Las inteligencias múltiples y el desarrollo personal. Montevideo. Uruguay: Cadiex Internacional. S.A.

Morin Edgar. UNESCO. (2003). Los siete saberes del futuro. Ecuador: Santillana.

Morin Edgar. (2003). Pensamiento complejo .

Posner, George J. (2004). Análisis del Currículo. México: McGraw Hill. Tercera Edición.

Tobón Sergio. (2006). Formación basada en competencias. Colombia: Ecoe Ediciones.

Tyler Ralph. W... (1973). Principios básicos del currículo. Buenos Aires: Troquel.

Salgueiro Amado. (s/a.). Planificación: El Arte de establecer Objetivos. Quito, Ecuador.

Enciclopedia General de la Educación. (1999). Barcelona. España: Grupo Editorial Océano.

Enciclopedia de la Psicología y Pedagogía. (1998). España: Océano-Centrum.

Problemas de aprendizaje. (1999). México: Ediciones Euroméxico, S.A. de C.V.

Sacristán J. Gimeno. (2002). Comprender y transformar la enseñanza. Morata. España.

Vigotsky L. S. (2004). Pensamiento y Lenguaje. Cuba: Editorial Pueblo y Educación.

Woolfolk Anita E. (1999). Psicología educativa. México: Printice Hall. Séptima Edición.

Wood, Thomas L y Brophy Jere. (1997). Psicología Educativa Contemporánea. México: McGraw Hill. Quinta Edición. 
